\title{
Disconnected Electromagnetic Form Factors
}

\author{
Walter Wilcox ${ }^{\mathrm{a}}$

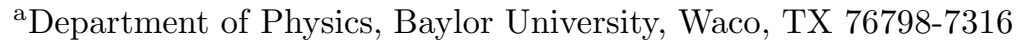

Preliminary results of a calculation of disconnected nucleon electromagnetic factors factors on the lattice are presented. The implementation of the numerical subtraction scheme is outlined. A comparison of results for electric and magnetic disconnected form factors on two lattice sizes with those of the Kentucky group is presented. Unlike previous results, the results found in this calculation are consistent with zero in these sectors.

\section{Introduction}

Fig.1 illustrates a quark "disconnected diagram" in lattice QCD. These are present in almost all hadron structure calculations and represent one of the greatest calculational challenges in the field of lattice QCD. Experimental results from Thomas Jefferson National Laboratory are now closing on on these small effects in the form of the strange quark contribution to the nucleon electromagnetic form factors.

The only previous published lattice results on electric and magnetic disconnected form factors was in a 1998 paper by Dong, Liu and Williams [1]. They found that after chiral extrapolation the disconnected parts gave

$$
\begin{gathered}
G_{M}^{s}(0)=-0.36 \pm 0.20, \\
\sqrt{\left|<r_{s}^{2}>_{E}^{n}\right|} \approx 0.25 \longleftrightarrow 0.4 \mathrm{fm} .
\end{gathered}
$$

The last number is from monopole fits to the electric disconnected data. (Measured total is $\left.\sqrt{\left|<r^{2}\right\rangle_{E}^{n}}=0.35 \pm 0.03 \mathrm{fm}\right)$.

$$
\text { HAPPEX [2] gives }
$$

$\left(G_{E}^{s}+0.392 G_{M}^{s}\right) /\left(G_{M} / \mu_{p}\right)=0.091 \pm 0.54 \pm 0.039$, at $q^{2}=0.477(\mathrm{Gev} / \mathrm{c})^{2}$. Similarly there is a less accurate number from SAMPLE[3] at $q^{2}=$ $0.1(\mathrm{Gev} / \mathrm{c})^{2}$ :

$G_{M}^{s}\left(.1(\mathrm{Gev} / \mathrm{c})^{2}\right)=0.61 \pm 0.17 \pm 0.21$.

Thus, experimentally there are no solid, separate numbers yet for disconnected $G_{M}^{s}\left(q^{2}\right)$ or $G_{E}^{s}\left(q^{2}\right)$.

Theoretically, results for these quantities from different models are in poor agreement [2]. Preliminary results for disconnected electric and

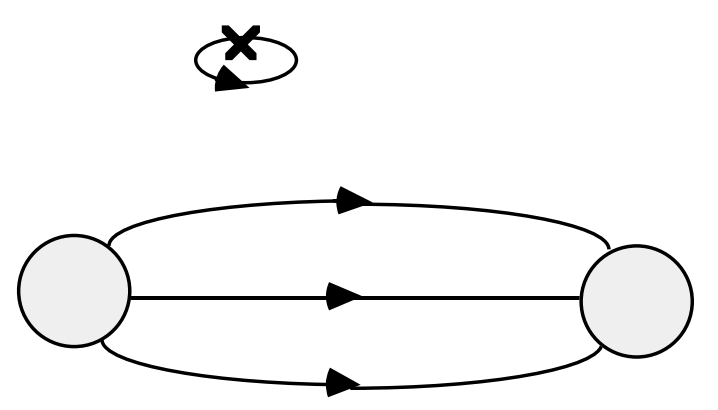

Figure 1. A generic nucleon disconnected diagram. The time axis is horizontal and the shaded circles represent nucleon sources or sinks.

magnetic form factors will be presented here for the case where the disconnected quark has the same mass as the valence quarks. In order to extract the strange quark contribution a chiral extrapolation of the different quark mass case must still be done.

\section{Noise and Subtraction Methods}

The basic method used to extract the signal is $Z 2$ noise 掼 with perturbative noise subtraction. Consider $Q$ such that

$$
<\operatorname{Tr}\{\tilde{Q} X\}>=0,
$$

where $X$ is the noise matrix. One then has of course for general $Q$

$<\operatorname{Tr}\{(Q-\tilde{Q}) X\}>=<\operatorname{Tr}\{Q X\}>$ 
However, the variance will not be the same,

$V[\operatorname{Tr}\{(Q-\tilde{Q}) X\}] \neq V[\operatorname{Tr}\{Q X\}]$.

The natural choice for $\tilde{Q}$, which must mimick the off-diagonal components of $Q$ to reduce the variance, is the perturbative expansion of the quark matrix [5]. This is given by

$M_{p}^{-1}=I+\kappa P+\kappa^{2} P^{2}+\kappa^{3} P^{3}+\cdots$.

where for Wilson fermions $(I, J=\{x, a, \alpha\})$

$$
\begin{aligned}
P_{I J}= & \sum_{\mu}\left[\left(1+\gamma_{\mu}\right) U_{\mu}(x) \delta_{x, y-a_{\mu}}+\right. \\
& \left.\left(1-\gamma_{\mu}\right) U_{\mu}^{\dagger}\left(x-a_{\mu}\right) \delta_{x, y+a_{\mu}}\right] .
\end{aligned}
$$

For a given operator, $\mathcal{O}$, the matrix $\mathcal{O} M_{p}^{-1}$ encountered in $\left\langle\bar{\psi} \mathcal{O} \psi>\rightarrow-\operatorname{Tr}\left(\mathcal{O} M_{p}^{-1}\right)\right.$ is not traceless. In other words, one must re-add the perturbative part, subtracted earlier, to get the full, unbiased answer.

Local operators require perturbative corrections starting at 4 th order in $\kappa$ (odd powers need no correction) and point-split ones require corrections starting at 3rd order (even powers need no correction). This procedure has been implemented exactly to 4th order (a single exact subtraction) for the point-split conserved vector current on the lattice for the lowest 4 nonzero lattice momentums.

\section{Analysis Method}

A new analysis method was used for the disconnected data analysis. At nonzero momentum, the basic quantity to consider is a ratio of nucleon three point and two point funxtions [1],

$R^{(e, m)}\left(t, t^{\prime}, \vec{q}\right) \equiv \frac{G^{(3 e, m)}\left(t^{\prime}, t, \vec{q}\right)}{G^{(2)}(t, 0)} \frac{G^{(2)}\left(t^{\prime}, 0\right)}{G^{(2)}\left(t^{\prime}, \vec{q}\right)}$,

where $G^{(3 e, m)}$ is the appropriate electric or magnetic three point function and $G^{(2)}(t, \vec{q})$ is the nucleon two point function extending from the time origin to $t$ with momentum $\vec{q}$. The three point function comes from the correlation of the two point function and the loop data. Calling this Fourier transformed loop data $L^{(e, m)}(\vec{q}, t)=$ $\sum_{\vec{x}} e^{-i \vec{q} \cdot \vec{x}} J(\vec{x}, t)$, where $J(\vec{x}, t)$ is the appropriate component of the lattice self-contracted current, then generically

$$
\begin{array}{r}
G^{(3 e, m)}\left(\vec{q}, t^{\prime}, t\right)=<G^{(2 e, m)}(t, 0) L^{(e, m)}\left(t^{\prime}, \vec{q}\right)> \\
-<G^{(2 e, m)}(t, 0)><L^{(e, m)}\left(t^{\prime}, \vec{q}\right)>,
\end{array}
$$

where the average is over configurations. The signal is always in the imaginary part of the lattice electromagnetic current $J(\vec{x}, t)$, which simplifies the analysis and improves error bars 88,96.

The actual measurement used here is,

$$
\begin{aligned}
& M^{(e, m)}\left(t-\frac{1}{2}, \vec{q}\right) \equiv \\
& \sum_{t^{\prime}=1}^{t+1}\left(R^{(e, m)}\left(t, t^{\prime}, \vec{q}\right)-R^{(e, m)}\left(t-1, t^{\prime}, \vec{q}\right)\right) .
\end{aligned}
$$

One can show for the appropriate components of $G^{(3 e, m)}\left(\vec{q}, t^{\prime}, t\right)$ and $L^{(e, m)}\left(t^{\prime}, \vec{q}\right)$ that $M^{(e, m)}(t-$ $\left.\frac{1}{2}, \vec{q}\right) \rightarrow G_{E}\left(\vec{q}^{2}\right), \quad \frac{q}{E+m} G_{M}\left(\vec{q}^{2}\right)$ for $t \gg 1$ in the electric or magnetic cases, respectively. The jackknife technique was used to define correlated error bars and the time range $t=10-12$, where the nucleon propagator becomes single exponential, was fit for all $\vec{q}$. The measurement in Eq.(8) is represented graphically in Fig.6.

The use of the same time-summed loop background in both terms in (8) leads to significantly smaller error bars. Eq.(8) implements the SESAM "plateau" strategy 10 by requiring the quark loop background to lengthen in time along with the nucleon propagators. Linear $t$ fits of the individual terms in (8) nicely avoid systematic errors coming from "off-shell" or contact terms $\left(t^{\prime} \sim 0\right.$ or $t^{\prime} \sim t$, constants for $t \gg 1$ [11]) since the loop time edges do not change their relation to the nucleon propagator time edges. However, the statistical errors are substantial. In contrast, the two terms in (8) could in principle possess different off-shell contributions because the $t^{\prime}$ sum in the second term includes additional correlations with loop data two time steps beyond the nucleon sink (see Fig.6). It has been explicitly verified that no discernable numerical correlations exist in such data, and so can be safely included.

\section{Results and Conclusions}

Two lattices sizes were used for comparison purposes: $20^{3} \times 32$ and $16^{3} \times 24$ at $\beta=6$.0. Re- 


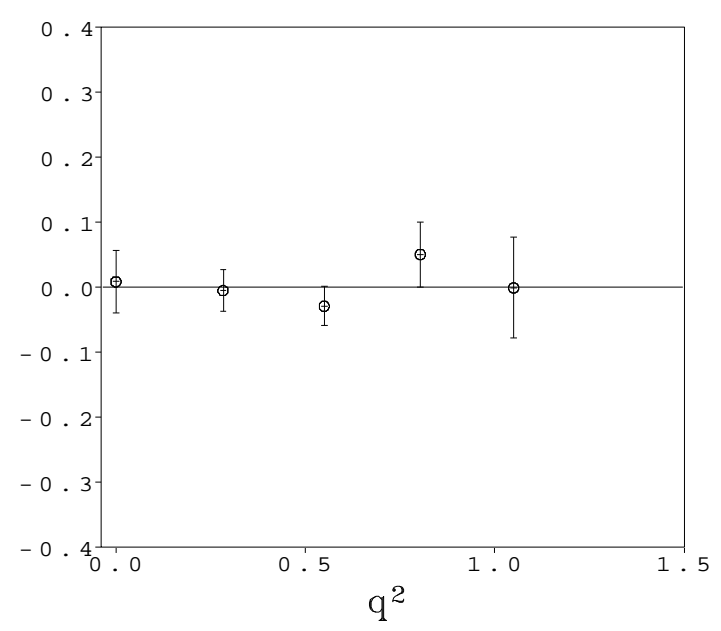

Figure 2. Disconnected nucleon electric form factor at $\kappa=0.152$ on the $20^{3} \times 32$ lattice as a function of $q^{2}$ in $(\mathrm{GeV} / \mathrm{c})^{2}$.

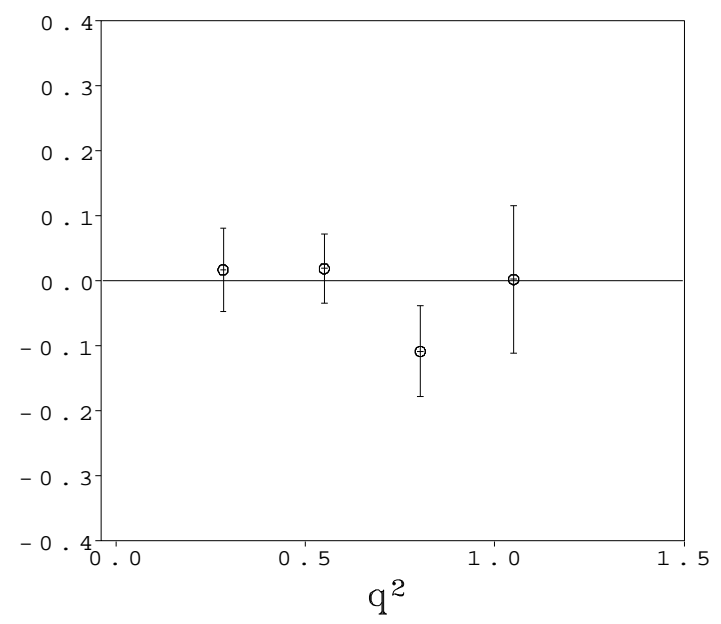

Figure 3. Same as Fig.2 but for the disconnected nucleon magnetic form factor.

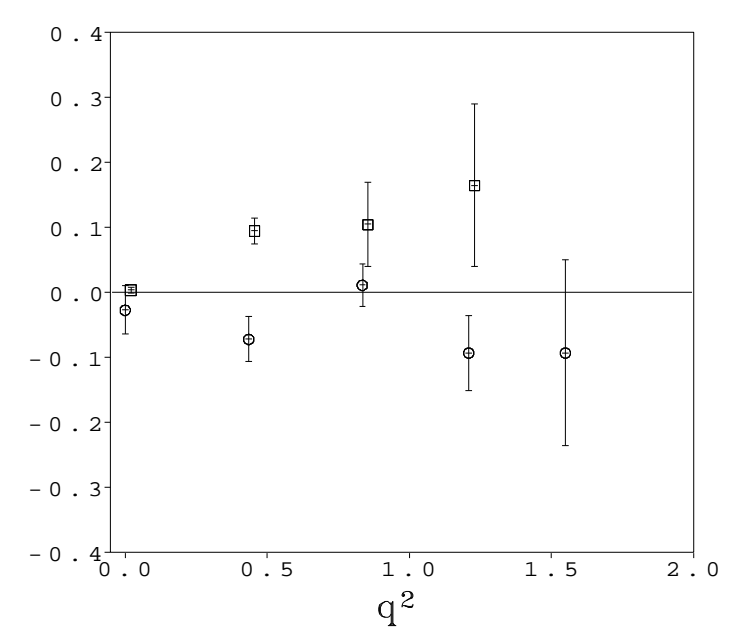

Figure 4. Disconnected nucleon electric form factor at $\kappa=0.152$ on the $16^{3} \times 24$ lattice as a function of $q^{2}$ in $(\mathrm{GeV} / \mathrm{c})^{2}$. Squares represent previous results from Ref.[1], circles are present results. Data here and in Fig. 5 separated in $q^{2}$ for better visibility.

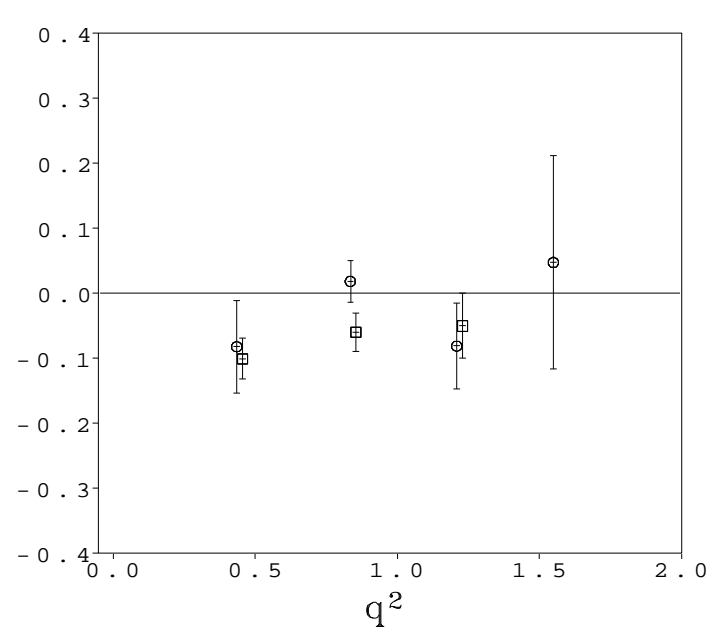

Figure 5. Same as Fig.4 but for the disconnected nucleon magnetic form factor. 


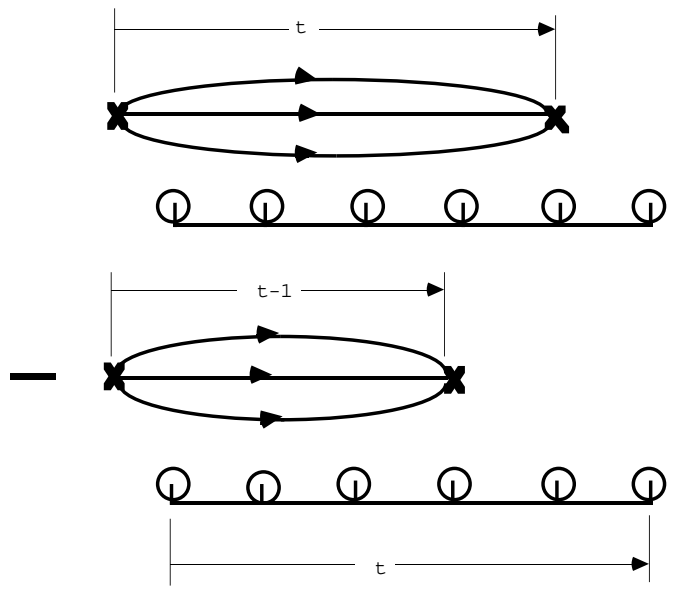

Figure 6. Illustrating the two terms in the disconnected amplitude measurement, Eq.(8). The time-summed quark loop data, symbolized by the underneath circles, is correlated with the appropriate nucleon amplitude and multiplied by a ratio of two point functions.

sults at $\kappa=0.152$ on both lattices will be examined here (both use 100 configurations). 30 noises on the $20^{3} \times 32$ lattice were used and 60 noises on the $16^{3} \times 24$ lattices. As has been shown previously [6] at fourth order subtraction for the electromagnetic current, one gains a factor of about 10 in the number of $Z 2$ noises at essentially zero overhead. Thus these results are equivalent to about 300 unsubtracted noises on the large lattices and about 600 unsubtracted noises on the small lattices.

Results are presented at $\kappa=0.152$ for the $20^{3} \times 32$ lattices in Figs. 2 and 3 above. At this $\kappa$ value there is no evidence for a signal in either the electric or magnetic sectors. In order to compare more directly with the results of Ref.[1], done on $10016^{3} \times 24$ lattices with 300 unsubtracted noises, the calculation was repeated on identically sized lattices. Again, there is no evidence of a signal at nonzero momentum in the electric or magnetic sectors in Figs.4 and 5. This finding is in disagreement with the Kentucky results[1,96]. The most serious disagreement is the electric sector, as can be seen in Fig.4.

The main message of these calculations to this point is that these correlations are extremely small QCD effects, both in the electric and magnetic sectors. It will be crucial to find better methods which will extract the signals in a more efficient way. Isolating these signals and making a definitive prediction will be one of the greatest challenges facing hadronic lattice QCD in the years to come.

\section{Acknowledgements}

This work is supported in part by NSF Grant Nos. 9722073 and 0070836 and NCSA and utilized the SGI Origin 2000 System at the University of Illinois. The author thanks S. J. Dong and K. F. Liu for their data in Figs.4 and 5.

\section{REFERENCES}

1. S. J. Dong, K. F. Liu, A. G. Williams, Phys. Rev. D58 (1998) 074504.

2. K. A. Aniol et. al., Jun 2000., WM-00107 (submitted to Phys. Rev. Lett.; nuclex/0006002).

3. D. T. Spayde et. al., Phys. Rev. Lett. 84 (2000) 1106.

4. S. Bernardson, P. McCarty and C. Thron, Comp. Phys. Comm. 78 (1994) 256.

5. C. Thron, S. J. Dong, K. F. Liu, H. P. Ying, Phys. Rev. D57 (1998) 1642.

6. W. Wilcox, Nucl. Phys. B (Proc. Suppl.) 8384 (2000) 834; W. Wilcox, "Noise Methods for Flavor Singlet Quantities", A. Frommer et. al. (eds.), Series Lecture Notes in Computational Science and Engineering, Springer Verlag, Heidelberg, 2000.

7. C. Michael, M. Foster and C. McNeile, Nucl. Phys. B (Proc. Suppl.) 83-84 (2000) 185.

8. W. Wilcox and B. Lindsay, Nucl. Phys. B (Proc. Suppl.) 63A-C (1998) 973.

9. M. Nilmani and S. J. Dong, these proceedings.

10. J. Viehoff et. al., Nucl. Phys. B (Proc. Suppl.) 63A-C (1998) 269.

11. R. Gupta et. al., Phys. Rev. D44 (1991) 3272. 